\title{
Do frontal dysfunctions play a role in visual hallucinations in Alzheimer's disease as in Parkinson's disease? A comparative study
}

\author{
Dario Grossi ${ }^{1,2}$, Anna Carotenuto ${ }^{3}$, Luigi Trojano ${ }^{1}$, Valentino Manzo ${ }^{4}$, and Angiola Maria Fasanaro ${ }^{4}$ \\ 1 - Second University of Naples, Caserta, Italy \\ 2 - Interuniversity Center for Research in Neurosciences, Caserta, Italy \\ 3 - Camerino University, Macerata, Italy \\ 4 - A.O. Cardarelli, Naples, Italy
}

\begin{abstract}
Recent studies have demonstrated that nondemented patients with Parkinson's disease with visual hallucinations had lower scores on frontal-executive tasks than parkinsonian patients without hallucinations, most likely due to defective cholinergic circuitry. The aim of the present study is to investigate whether development of visual hallucinations in patients with Alzheimer's disease may also be related to more severe frontal dysfunctions. In the present study, 36 patients were included who were affected by probable Alzheimer's disease (18 with visual hallucinations and 18 without) and 38 patients affected by idiopathic Parkinson's disease (19 with visual hallucinations and 19 without). Patients completed a neuropsychological test battery and a short questionnaire to collect information about hallucination types and features. Multivariate analysis showed that patients with Alzheimer's disease scored significantly lower than patients with Parkinson's disease and that patients with hallucinations scored significantly lower than patients without hallucinations. Within both the Alzheimer's disease group and the Parkinson's disease group, patients with visual hallucinations scored significantly lower than patients without visual hallucinations, particularly on tests evaluating frontalexecutive functions. These results demonstrate that patients with visual hallucinations show a significant impairment on tests tapping frontal-executive functions in Alzheimer's disease, as previously demonstrated (and verified here) in Parkinson's disease. On this basis it seems likely that analogous cognitive mechanisms underlie development of visual hallucinations in both degenerative diseases. Moreover, we may speculate that a defective circuitry of the prefrontal cortex is crucial for the genesis of hallucinations. Keywords: visual hallucinations, Parkinson's disease, Alzheimer's disease, frontal dysfunction.
\end{abstract}

Received 3 October 2011; received in revised form 25 November 2011; accepted 25 November 2011. Available online 29 December 2011

\section{Introduction}

Hallucinations were originally defined by Esquirol at the beginning of the 19th century as "a perception without an object." Hallucinations may involve any sensorial modality and are often reported in psychosis but also in degenerative brain pathologies such as Parkinson's disease (PD) and Alzheimer's disease (AD) (Fenelon, Mahieux, Huon, \& Ziegler, 2000). Visual hallucinations (VH), in particular, are quite common in PD patients, with a prevalence ranging from $8.8 \%$ to $44 \%$ (Barnes \& David,

Dario Grossi, Department of Psychology, Second University of Naples, Italy and Interuniversity Center for Research in Neurosciences, Caserta, Italy. Luigi Trojano, Second University of Naples, Caserta, Italy. Anna Carotenuto, Centro di Ricerche Cliniche, Telemedicina e Telefarmacia, Università di Camerino, Italy. Valentino Manzo and Angiola Maria Fasanaro, A.O. Cardarelli, Naples, Italy. Correspondence regarding this article should be directed to: Dario Grossi, Dept. of Psychology, Second University of Naples, Via Vivaldi 43, 81100 Caserta, Italy. E-mail: dario.grossi@unina2.it
2001). In nondemented PD patients with VH, more severe executive defects were found than in PD patients without VH. On this basis, it has been suggested that VH may be related to a specific dysfunction of prefrontal corticosubcortical circuits (Grossi et al., 2005b). Several studies supported this hypothesis (Collerton, Perry, \& McKeith, 2005; Ozer et al., 2007; Imamura, Wada-Isoe, Kitayama, \& Nakashima, 2008; Barnes, Boubert, Harris, Lee, \& David, 2003; Santangelo et al., 2007; Manganelli et al., 2009). Among others, a follow-up study (Santangelo et al., 2007) demonstrated that low scores on executive tasks may predict the onset of hallucinations and, in turn, that hallucinations and poor scores on executive tasks may predict development of dementia in PD patients. A more recent study (Manganelli et al., 2009) using transcranial magnetic stimulation combined with short-latency afferent inhibition confirmed that nondemented PD patients with VH had defective cholinergic circuitry and lower scores on executive tasks than PD patients without VH.

Executive functions are crucially involved in reality monitoring (Barnes \& Boubert, 2008) and contribute 
to disentangle visual perception, i.e., perception of external visual stimuli from imagination, i.e., internal production of a visual image (Grossi et al., 2005a; Roth, Johnson, Raye, \& Constable, 2009). A defective reality monitoring process may cause confusion between what is virtual and what is real, thus accounting for hallucinatory syndromes both in psychotic conditions and in neurodegenerative diseases (Brébion, Ohlsen, Pilowsky, \& David, 2008).

On the basis of the above considerations, we hypothesized that $\mathrm{VH}$ may be related to a marked deterioration of executive functions and prefrontal circuits in $\mathrm{AD}$ patients, analogous to what has been reported in PD patients. Although diminished activity of the cholinergic systems and of executive functions can be observed from the early stages of AD (Savioz, Leuba, Vallet, \& Walzer, 2009), it is possible that in some AD patients such defects are more severe, thus leading to $\mathrm{VH}$. If this were the case, $\mathrm{AD}$ patients with $\mathrm{VH}$ would show more severe impairments on executive tasks than AD patients without VH. The present study was aimed to test this prediction. For this purpose, we evaluated cognitive abilities, in particular performance on executive tests, in patients affected by AD with or without VH. Moreover, we also enrolled in the study PD patients with or without $\mathrm{VH}$ in order to compare cognitive correlates of $\mathrm{VH}$ in the two degenerative diseases.

\section{Materials and methods}

\section{Subjects}

We screened a consecutive series of outpatients affected by probable AD (identified according to standard diagnostic criteria) (McKhann et al., 1984) or by idiopathic Parkinson's disease (identified according to Parkinson's Disease Society Brain Bank criteria) (Gibb \& Lees, 1998). Patients were enrolled in the study if they met the following two inclusion criteria: disease duration of at least 2 years and at least 5 years of formal education. Exclusion criteria were as follows: severe dementia defined by a score on the Mini Mental State Examination (see below) $<15$, severe visual or auditory disturbances, and diagnosis of possible Lewy Body Disease according to standard clinical criteria (McKeith et al., 1996). Because major depression is known to be a confounding factor for neuropsychological disorders, we also excluded patients meeting DSM-IV criteria for current major depression and patients with relevant depressive signs and symptoms as expressed by a score $>16$ on the17-item Hamilton Rating Scale for Depression (Hamilton, 1960).

In all screened patients, presence of $\mathrm{VH}$ was searched for by means of a simple questionnaire that was completed by patients and their caregivers. Patients were selected for the $\mathrm{VH}$ group $(\mathrm{VH}+)$ if repeated occurrence of $\mathrm{VH}$ during the previous 3 months was reported concurrently by patients themselves and by their caregivers. On this basis, 18 patients affected by probable AD (11 females with a mean age of $72.8 \pm 8.0$ years; mean education $8.6 \pm 4.6$ years $)$, and 19 patients affected by idiopathic PD ( 8 females with a mean age of $68.3 \pm 8.3$ years; mean education $8.4 \pm 4.3$ years) were enrolled in the study.

From the same consecutive series of outpatients, we also selected a further sample of $18 \mathrm{AD}$ and $19 \mathrm{PD}$ patients who did not show $\mathrm{VH}$ on the questionnaires (VH-), fulfilled the same inclusion and exclusion criteria of $\mathrm{VH}+$ patients and matched $\mathrm{VH}+$ patients according to age and education.

All PD patients were treated with levodopa alone or with a combination of levodopa and a dopamineagonist (pramipexole, ropinirole or pergolide). All AD patients were treated with anticholinergic drugs (donepezil or rivastigmine). No patient demonstrated $\mathrm{VH}$ that were so severe and distressing as to require antipsychotic medications.

The study was approved by the local ethics committee. Written informed consent was obtained by all patients or by their caregivers.

\section{Neuropsychological testing}

All enrolled patients underwent a neuropsychological battery including screening tests for general cognitive abilities (Mini Mental State Examination, MMSE); Frontal Assessment Battery, FAB; Clock Drawing Test) and several tests for selected cognitive domains: constructional abilities (copying geometric drawings; immediate copying of the Rey complex figure); verbal and visuospatial learning (immediate and delayed recall of Rey 15 words; delayed reproduction of the Rey complex figure:); logical thinking (Raven's Coloured Progressive Matrices); frontal-executive functions: verbal fluency (semantic fluency test; phonemic fluency test); selective attention (attentional matrices), and inhibition of automatic responses (Stroop Color-Word test).

All tests were used in their Italian standardized version and were administered by the same trained psychologist, blind to patients' disease classification. A short questionnaire was administered to patients and their caregivers to gather information about type and features of $\mathrm{VH}$.

\section{Results}

Raw scores achieved by $\mathrm{AD}$ and $\mathrm{PD}$ patients enrolled in $\mathrm{VH}+$ and $\mathrm{VH}-$ groups are shown in Table 1. A two-way multivariate analysis of variance was performed on raw scores with disease (AD, PD) and presence of hallucinations $(\mathrm{VH}+, \mathrm{VH}-)$ as betweensubject independent variables. A significant effect of both main factors was found (disease: Wilks' lambda $=.483, p=.001$, partial eta squared $=.517$, observed 
Table 1. Mean scores on neuropsychological tests in $\mathrm{AD}$ and $\mathrm{PD}$ patients with $(\mathrm{VH}+)$ or without $(\mathrm{VH}-)$ visual hallucinations

\begin{tabular}{lllllllll}
\hline & \multicolumn{3}{c}{ AD patients $(\boldsymbol{n}=\mathbf{3 6})$} & \multicolumn{3}{c}{ PD patients $(\boldsymbol{n}=\mathbf{3 8})$} \\
\cline { 2 - 9 } & Mean & VH- & Mean & SD & Mean & SD & Mean & SD \\
\hline MMSE & 21.32 & 4.04 & 19.11 & 3.51 & 24.61 & 4.27 & 22.83 & 3.54 \\
Clock Drawing Test & 2.76 & 2.84 & 3.53 & 2.41 & 5.75 & 3.09 & 4.22 & 3.52 \\
FAB & 8.68 & 1.79 & 7.11 & 2.64 & 12.17 & 3.70 & $7.72^{\circ}$ & 3.34 \\
Copying drawings & 8.42 & 3.47 & 5.74 & 3.36 & 9.61 & 3.40 & 7.06 & 3.44 \\
Rey figure: immediate copying & 14.71 & 10.26 & 8.00 & 9.49 & 20.14 & 11.28 & 11.56 & 9.03 \\
Rey figure: delayed copying & 1.53 & 2.39 & .66 & 1.14 & 4.89 & 3.70 & 2.69 & 2.67 \\
15 words: immediate recall & 18.37 & 6.21 & $12.21^{*}$ & 6.40 & 24.44 & 8.73 & $17.06^{\circ}$ & 4.72 \\
15 words: delayed recall & 1.95 & 2.22 & .79 & .92 & 5.00 & 3.38 & 2.89 & 2.0 \\
Colored Progressive Matrices & 14.05 & 6.04 & 13.68 & 5.47 & 21.56 & 7.11 & 15.39 & 7.03 \\
Semantic fluency & 8.87 & 2.60 & 5.79 & 2.20 & 13.37 & 3.74 & $8.66^{\circ}$ & 3.04 \\
Phonemic fluency & 12.95 & 6.72 & $9.21^{*}$ & 6.01 & 19.44 & 9.87 & $9.17^{\circ}$ & 4.95 \\
Attentional matrices & 28.53 & 11.24 & 23.58 & 12.98 & 38.22 & 11.87 & $26.39^{\circ}$ & 12.53 \\
Stroop Color-Word test & 4.63 & 3.29 & $2.47^{*}$ & 2.82 & 7.72 & 7.68 & $3.61^{\circ}$ & 3.01 \\
\hline
\end{tabular}

MMSE: Mini Mental State Examination; FAB: Frontal Assessment Battery; AD, Alzheimer's disease; PD, Parkinson's disease; $\mathrm{VH}$, visual hallucinations; SD, standard deviation.

*Significantly lower than VH- AD.

${ }^{\circ}$ Significantly lower than VH- PD.

For all statistical comparisons significance threshold was set at $p<.0038$ according to Bonferroni's correction.

power $=.995 ;$ hallucinations: Wilks' lambda $=.517$, $p=.001$, partial eta squared $=.419$, observed power $=$ .990) without significant interaction between the two (Wilks' lambda $=.728, p=.094$, partial eta squared $=.272$, observed power $=.804$ ). Subsequent one-way multivariate analysis performed for $\mathrm{AD}$ and $\mathrm{PD}$ patients separately, with presence of hallucinations $(\mathrm{VH}+, \mathrm{VH}-)$ as a between-subject independent variable, showed that within the entire group of $\mathrm{AD}$ patients, the $\mathrm{VH}+$ group scored significantly lower than the $\mathrm{VH}-$ group (Wilks' lambda $=.231, p=.001$, partial eta squared $=.719$, observed power $=.996$ ), whereas within the entire group of $\mathrm{PD}$ patients $\mathrm{VH}+$ and $\mathrm{VH}-$ did not show overall significant differences (Wilks' lambda $=.459$, $p=.111$, partial eta squared $=.541$, observed power $=.768$ ) but significantly differed on the specific tests evaluating frontal functions. Comparisons among the single groups (with significance threshold set at $p<$ .0038 according to Bonferroni's correction for the high number of dependent variables) showed that $\mathrm{VH}+\mathrm{AD}$ and PD patients did not significantly differ on tests evaluating frontal functions (see Table 1), whereas $\mathrm{VH}+\mathrm{AD}$ patients scored significantly lower than $\mathrm{VH}+$ PD patients on MMSE $(p=.003)$, on delayed copying of the Rey figure, and on delayed recall of the Rey 15 words $(p=.000)$. Instead, VH- AD patients scored significantly lower than VH- PD patients on most neuropsychological scores. With respect to the presence of $\mathrm{VH}$ within $\mathrm{AD}$ and $\mathrm{PD}$ groups (see Table 1), $\mathrm{VH}+$ $\mathrm{AD}$ patients scored significantly lower than $\mathrm{VH}-\mathrm{AD}$ patients on the immediate recall of the Rey 15 words, on the phonemic fluency test and on the Stroop Color-Word test. Similarly, VH+ PD patients scored significantly lower than VH- PD patients on the immediate recall of the Rey 15 words on both the semantic and phonemic fluency tests on the test for selective attention and on the Stroop Color-Word test. Moreover, VH+ PD patients scored significantly lower than VH- PD patients on the screening test for frontal functions (FAB).

\section{Discussion}

The main findings of our study consist in the observation that performance on frontal-executive tests, such as the fluency tests and the Stroop test, was significantly lower in patients with $\mathrm{VH}$ than in patients without hallucinations, independent of neurological diagnosis. Another neuropsychological measure that showed the same pattern was immediate recall of the Rey 15 words that does not tap frontal-executive functions directly but, to a large extent, involves verbal working memory.

Phonemic (letter) fluency requires an efficient search strategy to select items from the phonological lexicon and is considered to involve the dorsolateral prefrontal cortex (Gaillard et al., 2000); verbal working memory is thought to be strongly dependent on supported dorsolateral prefrontal cortex as well. The Stroop test (interfered naming) explores selective attention and is considered to involve the prefrontal 
cortex, in particular the anterior cingulate cortex. From such considerations it is possible to infer that in both PD and $\mathrm{AD}$ groups occurrence of $\mathrm{VH}$ is particularly related to neuropsychological defects arising from dysfunction of the prefrontal cortex.

It is worth mentioning that in the PD group, $\mathrm{VH}+$ patients scored lower than $\mathrm{VH}-$ patients on the FAB (a screening battery for frontal dysfunction) and on attentional matrices, a further measure of selective attention. Therefore, in regard to $\mathrm{PD}$, the present results largely confirmed previous studies (e.g., Grossi et al., 2005b; Santangelo et al., 2009) showing an association between $\mathrm{VH}$ and neuropsychological defects on frontal tests. Data regarding AD patients have not been reported previously and, to the best of our knowledge, are novel. Multivariate analysis demonstrated that neuropsychological performance in the AD group was significantly lower than that observed in the PD group. From this finding it appears clear that, as expected, AD patients showed a more generalized cognitive impairment than PD patients. Yet, the present study would suggest that, even in presence of a general cognitive impairment, occurrence of $\mathrm{VH}$ may be related to more severe defects of frontal-executive functioning.

In the course of the cognitive decline in $\mathrm{PD}$ patients (Aarsland et al., 2004), presence of VH may herald dementia (Santangelo et al., 2007) and seems to be correlated with defective cholinergic circuitry of the prefrontal cortex (Manganelli et al., 2009). Based on such findings and in consideration of the present results, it would be possible to speculate that a prominent defect in prefrontal cholinergic neurotransmission may contribute to the genesis of hallucinations in a different degenerative disease, as is AD. This hypothesis should be tested further in studies directly tapping cholinergic transmission in AD patients with and without $\mathrm{VH}$.

The hypothesized relationships between occurrence of hallucinations and defects of prefrontal cortex would be in accordance with recent studies (Roth et al., 2009) showing that the left dorsolateral prefrontal cortex, the inferior frontal cortex bilaterally, and the left temporal cortex are involved in processes that allow to distinguishing visual perception "out of a subject" from visual experience "within a subject." In a further functional magnetic resonance study in subjects prone to hallucinate, $\mathrm{Ku}$ et al. (2008) identified a monitoring stage involving activation of the medial frontal cortex. These data are consistent with the neurofunctional model of hallucinations offered by Allen, Larøi, McGuire, and Aleman (2008), according to which the prefrontal cortex plays a fundamental role for genesis of $\mathrm{VH}$. The present clinical neuropsychological observation that, in both $\mathrm{PD}$ and $\mathrm{AD}, \mathrm{VH}$ are related to a defective performance on tests based on functioning of prefrontal cortex would further support such hypothesized role of the prefrontal cortex. It is well known that other brain dysfunctions may contribute to development of VH (see Vaphiades, Celesia, \& Brigell, 1996), but the present results would suggest that in $\mathrm{PD}$ and $\mathrm{AD}$ a defect of prefrontal monitoring systems seems strongly involved in generating "a perception without an object."

\section{References}

Aarsland, D., Andersen, K., Larsen, J. P., Perry, R., Wentzel-Larsen, T., Lolk, A., \& Kragh-Sørensen, P. (2004). The rate of cognitive decline in Parkinson disease. Archives of Neurology, 61(12), 1906-1911.

Allen, P., Larøi, F., McGuire, P. K., \& Aleman, A. (2008). The hallucinating brain: A review of structural and functional neuroimaging studies of hallucinations. Neuroscience and Biobehavioral Reviews, 32(1), 175-191.

Barnes, J., \& Boubert, L. (2008). Executive functions are impaired in patients with Parkinson's disease with visual hallucinations. Journal of Neurology, Neurosurgery and Psychiatry, 79(2), 190-192.

Barnes, J., \& David, A. S. (2001). Visual hallucinations in Parkinson's disease: A review and phenomenological survey. Journal of Neurology, Neurosurgery and Psychiatry, 70, 727-733.

Barnes, J., Boubert, L., Harris, J., Lee, A., \& David, A. S. (2003). Reality monitoring and visual hallucinations in Parkinson's disease. Neuropsychologia, 41(5), 565-574.

Brébion, G., Ohlsen, R. I., Pilowsky, L. S., \& David, A. S. (2008). Visual hallucinations in schizophrenia: Confusion between imagination and perception. Neuropsychology, 22(3), 383-389.

Collerton, D., Perry, E., \& McKeith, I. (2005). Why people see things that are not there: A novel Perception and Attention Deficit Model for recurrent complex visual hallucination. Behavioral and Brain Sciences, 28(6),737-757.

Fenelon, G., Mahieux, F., Huon, R., \& Ziegler, M. (2000). Hallucinations in Parkinson's disease. Prevalence, phenomenology and risk factors. Brain, 123, 733-745.

Gaillard, W. D., Hertz-Pannier, L., Mott, S. H., Barnett, A. S., Le Bihan, D., \& Theodore, W. H. (2000). Functional anatomy of cognitive development: fMRI of verbal fluency in children and adults. Neurology, 54(1), 180-185

Gibb, W. R., \& Lees, A. J. (1998). The relevance of the Lewy body to the pathogenesis of idiopathic Parkinson's disease. Journal of Neurology, Neurosurgery and Psychiatry, 51, 745-752.

Grossi, D., Imperati, F., Carbone, G., Maiorino, A., Angelillo, V., \& Trojano, L. (2005a). Visual and spatial positive phenomena in the neglected hemifield. A case report. Journal of Neurology, 252(6), 725-726.

Grossi, D., Trojano, L., Pellecchia, M. T., Amboni, M., Fragassi, N. A., \& Barone, P. (2005b). Frontal dysfunction contributes to the genesis of hallucinations in non-demented Parkinsonian patients. International Journal of Geriatric Psychiatry, 20(7), 668-673.

Hamilton, M. (1960). A rating scale for depression. Journal of Neurology, Neurosurgery and Psychiatry, 23, 56-62.

Imamura, K., Wada-Isoe, K., Kitayama, M., \& Nakashima, K. (2008). Executive dysfunction in non-demented Parkinson's disease patients with hallucinations. Acta Neurologica Scandinavica, $117(4), 255-259$.

Ku, J., Kim, J. J., Jung, Y. C., Park, I. H., Lee, H., Han, K., ... Kim, S. I. (2008). Brain mechanisms involved in processing unreal perceptions. NeuroImage, 43(4), 793-800.

Manganelli, F., Vitale, C., Santangelo, G., Pisciotta, C., Iodice, R., Cozzolino, A., ... Santoro, L. (2009). Functional involvement of central cholinergic circuits and visual hallucinations in Parkinson's disease. Brain, 132(9), 2350-2355

McKeith, I. G., Galasko, D., Kosaka, K., Perry, E. K., Dickson, D. W., Hansen, L. A., ... Perry, R. H. (1996). Consensus guidelines for the clinical and pathologic diagnosis of dementia with Lewy bodies (DLB): Report of the consortium on DLB international workshop. Neurology, 47(5), 1113-1124.

McKhann, G., Drachman, D., Folstein, M., Katzman, R., Price, D., \& Stradlan, E. M. (1984). Clinical diagnosis of Alzheimer's disease: Report of the NINCDS-ADRDA work group under the auspices of the Department of Health and Human Services task force on Alzheimer's disease. Neurology, 34, 939-944.

Ozer, F., Meral, H., Hanoglu, L., Ozturk, O., Aydemir, T., Cetin, S., 
Tiras, R. (2007). Cognitive impairment patterns in Parkinson's disease with visual hallucinations. Journal of Clinical Neuroscience, 14(8), 742-746.

Roth, J. K., Johnson, M. K., Raye, C. L., \& Constable, R. T. (2009). Similar and dissociable mechanisms for attention to internal versus external information. NeuroImage, 48(3), 601-608.

Santangelo, G., Trojano, L., Vitale, C., Ianniciello, M., Amboni, M., Grossi, D., \& Barone, P. (2007). A neuropsychological longitudinal study in Parkinson's patients with and without hallucinations. Movement Disorders, 22(16), 2418-2425.

Savioz, A., Leuba, G., Vallet, P. G., \& Walzer, C. (2009). Contribution of neural networks to Alzheimer disease progression. Brain Research Bulletin, 80(4-5), 309-314.

Vaphiades, M. S., Celesia, G. G., \& Brigell, M. G. (1996). Positive spontaneous visual phenomena limited to the hemianopic field in lesions of central visual pathways. Neurology, 47, 408-417. 